\title{
tic\&société
}

Vol. 15, $\mathbf{N}^{\circ} 1 \mid$ | er semestre $2021 \mid 2021$

Logique algorithmique et reproduction sociétale: les médiations sociales saisies par les algorithmes

\section{D’univocité en rétroaction : la critique de la cybernétique dans la pensée de Heidegger}

Unambiguous Meaning and Feedback Loops: Heidegger's Critique of Cybernetics Sobre la univocidad en retracción : la crítica de la cibernética en el pensamiento de Heidegger

\section{Baptiste Rappin}

\section{CpenEdition}

Journals

Édition électronique

URL : https://journals.openedition.org/ticetsociete/5698

DOI : 10.4000/ticetsociete.5698

\section{Éditeur}

Association ARTIC

Édition imprimée

Pagination : 41-67

Référence électronique

Baptiste Rappin, « D'univocité en rétroaction : la critique de la cybernétique dans la pensée de Heidegger », tic\&société [En ligne], Vol. 15, № 1 | 1er semestre 2021 | 2021, mis en ligne le 20 mai 2021 consulté le 22 mai 2021. URL : http://journals.openedition.org/ticetsociete/5698 ; DOI : https:// doi.org/10.4000/ticetsociete.5698 
D'univocité en rétroaction :

la critique de la cybernétique dans la pensée de Heidegger

\section{Baptiste RAPPIN}

baptiste.rappin@univ-lorraine.fr 


\section{D'univocité en rétroaction : \\ la critique de la cybernétique dans la pensée de Heidegger}

\section{Baptiste RAPPIN}

Baptiste Rappin est maître de conférences à l'IAE Metz School of Management (Université de Lorraine) et chercheur à l'Institut d'Histoire de la Philosophie (EA 3276, Aix-Marseille Université). II a publié, aux Éditions Ovadia, plusieurs ouvrages de philosophie du management dont, en 2014, Au fondement du Management, et en 2018, De l'exception permanente.

Résumé : Le concept d'information occupe une place importante dans l'œuvre du philosophe Martin Heidegger ; il s'agit alors pour nous de saisir cette opportunité pour mieux comprendre le mouvement de dé-médiation lié à l'expansion de la logique algorithmique. Nous observerons comment les fondements logiques de l'information liés à l'idéal d'univocité se trouvent convertis en boucle de rétroaction par la cybernétique : alors la régulation et le pilotage peuvent se substituer à l'institution comme nouvelle forme de gouvernementalité.

Mots-clés : Heidegger, cybernétique, information, rétroaction, univocité.

\section{Unambiguous meaning and feedback loops: Heidegger's critique of cybernetics}

Abstract: The concept of information is central to philosopher Martin Heidegger's work; we take advantage of this opportunity in order to better understand the movement of demediation in relation to the expansion of algorithmic logic. We observe how the logical foundations of information and the desire for unambiguous meaning are converted into the feedback loop by cybernetics, allowing regulation and management to replace institutions as a new form of governmentality.

Keywords: Heidegger, cybernetics, information, feedback loop, univocity. 


\section{D’univocité en rétroaction :}

la critique de la cybernétique dans la pensée de Heidegger

Sobre la univocidad en retracción: la crítica de la cibernética en el pensamiento de Heidegger

Resumen: El concepto de información ocupa un lugar fundamental en la obra del filósofo Martin Heidegger. Esto permite interpretar mejor el movimiento de de-mediación que la expansión de la lógica algorítmica implica. Se investiga el proceso a través del cual los fundamentos lógicos de la información relacionados con el ideal de univocidad se convierten en un bucle de retroalimentación cibernético: de esta forma, la regulación y la dirección pueden sustituir a la institución como una nueva forma de gobernanza.

Palabras clave: Heidegger, cibernética, información, retroalimentación, univocidad. 


\section{Baptiste RAPPIN}

L'algorithme, le projet d'une langue universelle, c'est la révolte contre le langage donné. On ne veut pas dépendre de ses confusions, on veut le refaire à la mesure de la vérité, le redéfinir selon la pensée de Dieu, recommencer à zéro l'histoire de la parole, ou plutôt arracher la parole à l'histoire (Merleau-Ponty, 1969, p. 10).

\section{Introduction : I'information, du bavardage au calcul}

Pour surprenante, voire paradoxale qu'elle puisse paraître, l'affirmation selon laquelle le philosophe Martin Heidegger prêta attention à la notion d'information tout au long de son œuvre n'en est pas moins vraie. Non sans quelque anachronisme, Jacques Derrida l'affirma lui-même à sa manière en déclarant à Dominique Janicaud (2001, p. 122) que le philosophe de Fribourg " a parlé de cybernétique toute sa vie ». On connaît en effet Heidegger pour l'analytique existentiale de Sein und Zeit, on retient de lui le tournant vers l'histoire de l'être, on fait de lui l'un des critiques de la Technique sous la forme de l'Arraisonnement planétaire et de la mise à disposition de l'étant, on étudie ses liens ambigus au régime national-socialiste débats accentués depuis la parution desdits Cahiers noirs-, mais on omet très souvent de revenir à ce fil directeur plus discret mais pourtant consistant: celui de l'information (Brown et Lightfood, 1998 ; Introna, 2001 ; Rappin, 2015).

Certes, le mot même d'information n'apparaît que tardivement, dans Le principe de raison, paru en 1957, et comprenant le cours du semestre d'hiver 1955-1956 ainsi que deux conférences prononcées en 1956. Le philosophe en donne la définition suivante, qui permet au lecteur d'en saisir d'emblée toutes les dimensions constitutives:

Information désigne d'abord une communication de nouvelles, aussi rapide, complète, claire et abondante que possible et qui renseigne l'homme d'aujourd'hui sur la mise en sûreté de ses besoins, des quantités qu'ils requièrent et des sources d'approvisionnement. II en résulte que la conception qui fait du langage un instrument d'information s'impose toujours davantage. Car c'est la définition du langage comme d'un moyen d'information qui seule a fourni la raison suffisante sur 


\section{D'univocité en rétroaction :}

\section{la critique de la cybernétique dans la pensée de Heidegger}

laquelle repose la construction des machines à penser et des grandes machines à calculer. Mais, en même temps que l'information informe, c'est-à-dire renseigne, elle in-forme, c'est-à-dire dispose et dirige. L'information, en tant que transmission de nouvelles, est donc aussi le dispositif qui donne à l'homme, à tous les objets et à tous les fonds, une forme telle qu'elle suffise à assurer la domination de l'homme sur la terre et même au-delà de la terre. (Heidegger, 1962, p. 260)

Trois étapes structurent le raisonnement de Heidegger, qui le conduisent à définir l'information. Dans un premier temps, l'information est assimilée à une "communication de nouvelles », propos qui renvoie aux paragraphes 35 ("Le ondit »), 36 ("La curiosité ») et 37 ("L'équivoque ») de Sein und Zeit (1986): le philosophe y expose la façon qu'a le Dasein (terme par lequel Heidegger désigne l'homme en tant qu'il existe) de se reposer sur des certitudes établies et de se fermer ainsi à une entente authentique du monde. Deuxième temps: l'information, qui désormais absorbe toutes les possibilités du langage, se transmet d'autant plus facilement qu'elle prend la forme univoque du calcul; ce caractère mathématique lui confère un pouvoir clarificateur à l'origine directe de la gestion des biens. Enfin, troisième et dernier temps: la puissance calculatoire de l'information, démultipliée par le progrès technique et machinique, en fait le vecteur d'un pouvoir de pilotage et de maîtrise de l'étant.

Dans le cadre de cet article, nous nous limiterons à l'analyse des deux derniers temps. Pourquoi ? Parce qu'ils éclairent de façon radicale, c'est-à-dire en remontant à la racine du phénomène, le mouvement général de «transformation digitale " de la société contemporaine. Les éclairages ontologique et généalogique qu'apporte Heidegger sur le concept d'information, qui n'est autre que la réduction du langage à la donnée, permettent de mieux saisir les enjeux sousjacents de la logique algorithmique. Nous serons alors plus particulièrement à même de mettre en exergue le lien direct entre, d'une part, la réduction de la langue à l'univocité opérée par la logique propositionnelle et, d'autre part, le procès général de dé-médiation engendré par la multiplication des boucles de rétroaction. 


\section{La place du calcul dans l'histoire de l'être}

En 1959, dans un texte intitulé Sérénité, Heidegger introduit la dichotomie entre la pensée qui calcule et la pensée méditante :

[...] lorsque nous dressons un plan, participons à une recherche, organisons une entreprise, nous comptons toujours avec des circonstances données. Nous les faisons entrer en ligne de compte dans un calcul qui vise des buts déterminés. Nous escomptons d'avance des résultats définis. Ce calcul caractérise toute pensée planifiante et toute recherche. Une pareille pensée ou recherche demeure un calcul, là même où elle n'opère pas sur des nombres et n'utilise ni simples machines à calculer ni calculatrices électroniques. La pensée qui compte calcule. Elle soumet au calcul des possibilités toujours nouvelles, de plus en plus riches en perspectives et en même temps plus économiques. La pensée qui calcule ne nous laisse aucun répit et nous pousse d'une chance à la suivante. La pensée qui calcule ne s'arrête jamais, ne rentre pas en elle-même. Elle n'est pas une pensée méditante, une pensée à la poursuite du sens qui domine dans tout ce qui est. (Heidegger, 1990a, p. 136)

Calculer, c'est tout d'abord compter avec les circonstances, en d'autres termes s'adapter à des facteurs de contingence; calculer, c'est ensuite planifier une activité en vue d'un résultat ; calculer, c'est enfin un cycle permanent qui ne connaît pas le repos. Le projet mathématique ne doit néanmoins pas seulement s'entendre de façon factuelle ou événementielle, comme l'arrivée de nouvelles techniques de calcul ou comme la réalisation de progrès dans l'ordre de la computation: c'est que, au-delà de ces innovations, il caractérise la modernité en son essence même. On connaît la fameuse métaphore de Galilée pour qui l'univers est un livre écrit en langue mathématique et géométrique. Et l'on sait que Newton eut simplement besoin de croire en l'existence de forces mathématiques, et non pas physiques, pour formuler les lois de l'attraction et de la gravité. Toutefois, comme l'écrit Heidegger, 


\section{D'univocité en rétroaction :}

\section{la critique de la cybernétique dans la pensée de Heidegger}

la fondation de la géométrie analytique par Descartes, celle du calcul des fluxions par Newton, la fondation à la même époque du calcul différentiel par Leibniz, toute cette nouveauté, tout ce mathématique au sens étroit, ne devint possible et même indispensable qu'en raison du trait mathématique fondamental de la pensée en général. (Heidegger, 1971a, p. 104)

La mathématique est une représentation fondamentale des choses qui se pose comme norme a priori de l'étant, il structure l'appréhension du monde, des objets comme des hommes. Pour Heidegger, le projet d'arraisonnement du monde provient de l'époque moderne qui, à partir de Descartes, confère au sujet une suprême souveraineté sur l'objet conduisant à sa manipulation, voire à sa transformation, dans l'omission de sa nature propre. Réduit à une étendue ainsi qu'à un ensemble de mécanismes, placé sous l'empire de l'âme et de sa volonté, le réel peut alors se prêter à toutes sortes d'aventures démiurgiques, telles que sa réécriture en langage mathématique ou sa mobilisation artificielle pour les besoins de l'expérimentation.

La pensée calculante prend sa source dans le principe de raison. Si ce dernier fut énoncé sous sa forme canonique - nihil est sine ratione - par Leibniz à la charnière des XVII et XVIII siècles, Heidegger (1962, p. 45-46) considère qu'il fut en état d'incubation depuis la naissance de la philosophie, passant ainsi plus de 2300 ans dans l'ombre avant de monter sur l'estrade et d'occuper le devant de la scène. "Rien n'est sans raison », cela signifie que tout ce qui existe, tout ce qui est, possède une raison d'exister et d'être, une raison que l'on peut rendre (principium reddandae rationis) : l'étant, comme objet, doit fournir à l'homme, comme sujet, sa raison, il doit rendre gorge.

La formulation du principe en langue latine est révélatrice : en effet, ratio désigne, avant le jugement et l'intelligence, le calcul mais aussi les relations d'affaires comme nous l'apprend le Gaffiot (2000, p. 1331) ; une étymologie que Heidegger emploie dans sa qualification de l'homme rationnel :

L'homme en conséquence est l'animal rationale, l'être vivant qui réclame des comptes et qui rend des comptes. L'homme, suivant cette définition, est l'être 
vivant qui compte : "compter" étant ici entendu au sens large que le mot ratio - d'abord un terme du parler commercial des Romains - prenait déjà chez Cicéron, à l'époque où la pensée grecque a été traduite en conceptions romaines. (Heidegger, 1962, p. 269)

Pourquoi Leibniz? Le philosophe allemand procède à un élargissement du tournant subjectif cartésien par l'introduction d'une monadologie, d'une science de la monade: par " monade », il faut entendre une substance simple, indivisible et éternelle qui possède un dynamisme interne, l'appétition, par lequel elle passe de perception obscure en perception claire. Les êtres humains sont des monades douées de raison et possèdent, contrairement aux animaux qui ne peuvent qu'ajouter la mémoire à la perception, les vérités nécessaires et éternelles par l'application de deux grands principes, celui de contradiction et celui de raison suffisante (Leibniz, 1995, p. 100-101). La ratio sufficiens, cette raison qui suffit et se suffit, est suprême (elle est Dieu chez Leibniz) car elle rend compte de l'univers par le calcul ; Heidegger écrit ainsi :

En 1677 (à l'âge de 31 ans), Leibniz écrivit un dialogue sur la lingua rationalis, c'est-à-dire sur le calcul, le mode de compte, qui devait permettre de calculer complètement et universellement, pour tout ce qui est, les relations entre le mot, le signe et la chose. Dans ce dialogue et dans d'autres œuvres, Leibniz a pensé et posé d'avance les fondements de ce qui, aujourd'hui, non seulement est utilisé sous forme de machines à penser, mais surtout détermine notre mode de penser. (Heidegger, 1962, p. 220)

Dans ce passage, Heidegger suggère un lien entre l'entreprise philosophique de Leibniz et le projet de machine à calculer universelle du mathématicien Alan Turing (1995, p. 66) qui apparaît alors, de ce point de vue, comme un accomplissement: "II est possible d'inventer une unique machine $U$ utilisable pour calculer n'importe quelle séquence calculable ». La machine est équipée d'un déchiffreur qui lit une bande infiniment longue; cette dernière est divisée en carrés identiques qui restent blancs ou sont barrés d'un trait oblique. La machine peut opérer quatre mouvements sur la bande: aller 


\section{D'univocité en rétroaction :}

\section{la critique de la cybernétique dans la pensée de Heidegger}

vers la droite et vers la gauche, effacer ou imprimer la barre oblique. Munie de ces opérations élémentaires, elle peut exécuter n'importe quel programme exprimable en code binaire. Quelques années plus tard, Norbert Wiener (2014a, p. 222) se montrera fidèle au projet de Turing :

C'est pourquoi la machine à calculer doit être logique autant qu'arithmétique, et doit combiner les éventualités selon un algorithme systématique. Là où de nombreux algorithmes pourraient être utilisés, le plus simple d'entre eux est connu comme l'algèbre de la logique par excellence, l'algèbre booléenne. Cet algorithme, comme l'arithmétique binaire, est basé sur la dichotomie, le choix entre oui et non, entre appartenir à une classe et ne pas lui appartenir.

L'anthropologie de la modernité, qui culmine dans le principe de raison chez Leibniz, fait alors de la logique le seul et unique mode de projection de l'homme dans le monde, conduisant à l'effondrement de la pensée qui tombe dans l'ignorance de son rapport originaire à l'être :

La primauté de l'Être travaille à l'oubli de l'Être. Par cette primauté le rapport spécifique à l'Être se trouve enseveli, qu'il convient de rechercher dans la pensée justement conçue. Requis par l'étant, l'homme en vient à tenir le rôle de l'étant normatif. En tant que rapport à l'étant, la connaissance lui suffit, laquelle doit nécessairement se résoudre dans l'objectivation et ainsi relever du calcul, conformément au genre d'essence de l'étant dans le sens du réel, assuré parce que planifiable. La promotion de la logistique au rang de la logique est signe de l'abaissement de la pensée. La logistique revient à organiser l'ignorance absolue quant à l'essence de la pensée, admis que la pensée, conçue essentiellement, est ce savoir qui projette, lequel, dans la sauvegarde de l'essence de la vérité, s'épanouit à partir de l'Être. (Heidegger, 1971b, p. 396)

Bien sûr, par "logistique », il faut entendre ici cette forme particulière de logique qui se développa au début du $X X^{e}$ siècle et qui, par le recours aux formalismes mathématiques, fomenta le projet de la création d'un langage absolument univoque. 


\section{Baptiste RAPPIN}

Remontons alors à présent aux fondements logiques du concept d'information.

\section{Les fondements logiques de l'information}

En effet, si l'information est communication de nouvelles, de renseignement, voire de propagande, elle est également le fruit d'un inlassable effort de formalisation de la langue ; c'est ce que note Heidegger en 1959 :

La parole ainsi mise en position devient information. Elle s'informe sur elle-même, afin d'assurer sa propre démarche par des théories informatiques. Le Dis-positif, déploiement partout régnant de la technique moderne, se rend disponible la langue formalisée, genre de l'information par la force de laquelle l'être humain se voit formé au déploiement technique et calculateur, c'est-à-dire installé en lui, abandonnant peu à peu la "langue naturelle". [...] Car la "langue naturelle", dont on doit encore parler, est d'emblée mise en jeu comme la langue non encore formalisée, mais promise à la formalisation. (Heidegger, 1976, p. 252)

À suivre Françoise Dastur (2007, p. 19-48), Heidegger s'empara de la question de la logique alors même qu'il était encore étudiant en théologie : cet intérêt soutenu donna lieu à la Dissertation de 1913, Die Lehre vom Urteil im Psychologismus (La théorie du jugement dans le psychologisme), qui sera suivie en 1915 de la thèse d'habilitation, Die Kategorien- und Bedeutungslehre des Duns Scotus (La théorie des catégories et de la signification chez Duns Scot). Enthousiasmé par la lecture des Recherches Logiques de Husserl, le jeune philosophe se méfie aussi bien du psychologisme, qui voudrait réduire le jugement à un processus psychique, que de la logistique, qui procède sinon à l'éradication du moins à l'appauvrissement du sens : à l'écart de ces deux voies sans issue, Heidegger conçoit la logique comme l'étude des structures d'articulation des signes ayant un sens.

Plus précisément, Heidegger entrevoit, à son époque, l'hégémonie de ce que l'on nommera bien plus tard « philosophie analytique $»$ : 


\section{D'univocité en rétroaction :}

\section{la critique de la cybernétique dans la pensée de Heidegger}

C'est seulement tout récemment qu'on a fait fructifier scientifiquement ces connaissances de la logique, et ce dans une science particulière qui se nomme "logistique". Elle est la plus spéciale de toutes les sciences spéciales. La logistique passe actuellement en nombre d'endroits, avant tout dans les pays anglo-saxons, pour la seule forme possible de philosophie stricte, parce que ses résultats et ses méthodes sont d'un rapport sûr et immédiat pour la construction du monde technique. La logistique commence ainsi actuellement, en Amérique et ailleurs, à établir son emprise sur l'esprit, comme étant la véritable philosophie de l'avenir. (Heidegger, 1999, p. 33-34)

Tentons de comprendre comment le philosophe en vient à formuler ce jugement. Un petit détour historique s'avère ici expédient.

La logique aristotélicienne est une logique des termes qui consiste, par le truchement de la copule, à attribuer un prédicat à un sujet. Le raisonnement, à l'origine d'un nouveau jugement, culmine dans le syllogisme, dont l'exemple le plus connu est le suivant : "Tous les hommes sont mortels ; or tous les Grecs sont des hommes ; donc tous les Grecs sont mortels ». Le syllogisme enchaîne trois termes à travers une prémisse majeure, contenant le moyen terme universel comme sujet (" hommes " dans l'exemple canonique) et une prémisse mineure, contenant le moyen terme comme prédicat, qui débouchent sur la conclusion qui met en relation le grand terme (« mortels ») et le petit terme ("Grecs »). Cette logique est formelle puisque le raisonnement débouche nécessairement sur la conclusion; en revanche, elle n'est guère formaliste, comme elle le deviendra aux Temps modernes, car elle porte sur des contenus et non sur des signes abstraits : en d'autres termes, la vérité formelle ne peut s'émanciper de la vérité matérielle.

La logique moderne, quant à elle, réside dans le passage décisif d'une logique des termes à une logique propositionnelle. Si l'on fait généralement du logicisme de Frege le lieu de ce tournant, il ne faut pas oublier que les stoïciens posèrent déjà la proposition comme l'atome de la logique, innovation qui fut 
éclipsée par le succès de la pensée aristotélicienne ; ni, non plus, Pierre Abélard ou encore Guillaume d'Ockham, dont l'affûté rasoir, principe d'économie s'il en est, tranchait tout lien qui osait relier la logique à la métaphysique; ni, enfin, Leibniz qui désirait accoucher d'une langue artificielle (idéographique et non phonétique) émancipée des contraintes par trop restrictives des langues naturelles.

Frege mit au point une écriture symbolique nouvelle, qu'il baptisa " idéographie », avec l'objectif affiché qu'aucun signe ne pût posséder plusieurs sens. Ce faisant, l'arithmétique devint une extension, ou un point d'application, de la logique élevée au rang de discipline universelle : "Le langage par formules de l'arithmétique est une idéographie puisqu'il exprime immédiatement la chose sans passer par les sons " (Frege, 1971, p. 68). D'où le corollaire suivant : les sujets et les prédicats sont évacués et laissent place à la fonction et à son argument ; ainsi « Socrate est mortel » devient $\mathrm{f}$ (Socrate) dont le résultat, binaire, est soit vrai soit faux. S'ensuivent la définition d'opérateurs logiques (comme le conditionnel ou la négation) puis l'introduction de quantificateurs (universel et existentiel) qui dépouillent la langue de toutes ses scories pour édifier un calcul propositionnel fondé sur une syntaxe rigoureuse. L'ambition de Frege fut ainsi d'édifier un symbolisme parfait et d'en dégager les lois internes, que l'auteur nomme "fondamentales », qui dictent les procédés de transformation, de déduction et d'inférence des propositions. Son objectif fut de parvenir à une sémantique fondée sur l'univocité : à chaque proposition une seule et unique référence. C'est bien là que réside la raison d'être de son entreprise : en effet, si Frege se lance dans ce gigantesque projet de l'idéographie, c'est bien pour pallier les lacunes du langage: "[...] le langage se révèle défectueux lorsqu'il s'agit de prévenir les fautes de pensée. II ne satisfait pas à la condition ici primordiale, celle d'univocité » (Frege, 1971, p. 64). D'où la nécessité, ajoute le philosophe un peu plus loin, de recourir à « un ensemble de signes, purifiés de toute ambiguïté, et dont la forme strictement logique ne laisse pas échapper le contenu » (Frege, 1971, p. 66).

L'univocité peut être considérée comme la bannière de rassemblement de la logique propositionnelle moderne, qui prendra plus tard le nom de " philosophie analytique ». Prenons 


\section{D'univocité en rétroaction :}

\section{la critique de la cybernétique dans la pensée de Heidegger}

les exemples suivants, dont les auteurs sont les contemporains de Heidegger.

Russell se lança, en compagnie d'Alfred North Whitehead, dans l'aventure des Principia Mathematica. Prenant acte de l'axiomatisation généralisée des différents pans des mathématiques, les auteurs proposèrent de réduire la notion mathématique d'ensemble, au fondement des travaux de Georg Cantor, à la dimension logique de fonction propositionnelle. Ils élaborèrent alors, à l'instar de Frege, une idéographie à partir de laquelle ils pouvaient fonder les théorèmes de la logique des prédicats, des classes puis des relations.

L'univocité est ensuite le fer de lance du Cercle de Vienne dans sa lutte contre la métaphysique et ses profondeurs :

De là, la recherche d'un système formulaire neutre, d'un symbolisme purifié des scories des langues historiques, de là aussi la recherche d'un système total de concepts. La netteté et la clarté sont visées, les lointains sombres et les profondeurs insondables refusés ; en science, pas de "profondeurs", tout n'est que surface. [...] Tout est accessible à l'homme, et l'homme est la mesure de toutes choses. Ici la parenté avec les sophistes, non avec les platoniciens, devient évidente [...]. La conception scientifique du monde ne connaît pas d'énigmes insolubles. (Hahn, Neurath et Carnap, 2010, p. 110-111)

Enfin, le jeune philosophe Ludwig Wittgenstein s'inscrit bien dans la lignée de la logique propositionnelle dont il livrait les principes à travers une série d'aphorismes: " Je conçois la proposition - avec Frege et Russell - comme fonction des expressions qu'elle contient » (Wittgenstein, 1993, 3.318, p. 46). Sous sa plume, toute philosophie devient logique, c'est-à-dire analyse et recréation d'un langage symbolique seul à même, dans un choix qui se réduit encore à l'alternative binaire, de décrire la réalité :

La réalité doit être fixée par oui ou par non grâce à la proposition.

II faut pour cela qu'elle soit complètement décrite par la proposition. 
La proposition est la description d'un état de choses. (Wittgenstein, 1993, 4.023, p. 53)

Univocité, voici justement le trait par lequel Heidegger caractérise l'information :

La série des signes se trouve reconduite à une série de décisions oui-non. Des machines sont commises à la production de telles séries : celles-ci, grâce aux flux de courant et aux impulsions électriques, suivent ce modèle abstrait de production de signes et fournissent les messages correspondants. Pour qu'une telle espèce d'information devienne possible, chaque signe doit être défini de façon univoque; de même chaque ensemble de signes doit signifier de façon univoque un énoncé déterminé. L'unique caractère de la langue qui subsiste dans l'information est la forme abstraite de l'écriture, qui est transcrite dans les formules d'une algèbre logique. L'univocité des signes et des formules qui est nécessairement exigée de ce fait assure la possibilité d'une communication certaine et rapide. (Heidegger, 1990b, p. 38-39)

De façon plus concise, Heidegger cerne le cœur de la révolution logique moderne dans le fait que la proposition, et non plus le terme, devienne la brique élémentaire du raisonnement : "Elle se réduit à la proposition: la langue est information » (Heidegger, 1990b, p. 35). En convertissant la parole en information, geste qui relève selon le philosophe d'une " agression », et même de " l'agression la plus violente et la plus dangereuse " (Heidegger, 1990b, p. 40), la technique moderne constitue "une menace contre l'essence la plus propre de l'homme » (1990b, p. 41). Pourquoi ? Pour Heidegger, qui reste ici d'une certaine façon phénoménologue, la langue est un dire qui montre la chose qui apparaît ou, encore mieux dit, un dire qui porte la chose au paraître. La langue est par conséquent une monstration, et non pas une démonstration qui repose sur la production de signes. L'univocité porte ainsi atteinte non seulement à la relation de l'homme à l'étant qui l'entoure, mais également à l'essence même de l'homme dans la mesure où il 


\section{D'univocité en rétroaction :}

\section{la critique de la cybernétique dans la pensée de Heidegger}

est cet étant pour lequel il y a des étants dont la manifestation est étroitement liée à la parole.

Mais franchissons un pas supplémentaire, car il convient de noter que cette révolution logique serait restée une affaire théorique, et presque innocente, si elle ne s'était pas incarnée dans l'informatique et les systèmes artificiels. Et c'est bien la conversion de l'univocité en boucle de rétroaction, opérée par la cybernétique, qui va doter la machine d'une puissance inégalable.

\section{La matrice cybernétique: de l'univocité à la rétroaction}

Assurément, Norbert Wiener, personnage central de l'histoire de la cybernétique, demeura fidèle à cette veine antimétaphysique du néo-positivisme; en effet, selon lui, « des mots comme la vie, les fins, l'âme sont grossièrement inutiles pour exprimer une pensée scientifique » (Wiener, 2014b, p. 63). Néanmoins, c'est bien la théorie des types logiques de Russell, dont il fut pendant deux ans l'étudiant à Cambridge (après son doctorat), qui exerça sur lui une profonde influence. De quoi s'agit-il ?

Distinguant les prédicats qui peuvent être prédiqués d'euxmêmes et ceux qui ne le peuvent pas, le philosophe britannique posa alors la question qui allait lui ouvrir le chemin de la théorie des types : le prédicat « prédicat qu'on ne peut prédiquer de luimême " peut-il être prédiqué de lui-même ? En termes moins techniques, ce paradoxe est connu pour être celui du menteur, déjà formulé par le philosophe mégarique Eubulide qui place les mots suivants dans la bouche d'Épiménide le Crétois : "Tous les Crétois mentent tout le temps ". S'il dit vrai, alors, en tant que Crétois, il ment en nous le disant ; s'il ment, il vérifie le contenu de sa proposition, énonçant alors la vérité. Pour sortir de ce cercle vicieux, Russell choisit de restreindre l'utilisation des symboles d'appartenance $(\in)$ et d'inclusion $(c)$ de telle manière qu'un objet de type $\mathrm{n}$ ne puisse appartenir qu'à un objet de type $\mathrm{n}+1$ : ainsi émergent des niveaux $\mathrm{n}$ de vérité contenant des propositions d'ordre $\mathrm{n}$. "Tous les Crétois mentent tout le temps » est une proposition de type 1, "Épiménide le Crétois dit: "Tous les Crétois mentent tout le temps" " est une 


\section{Baptiste RAPPIN}

proposition d'ordre 2. Russell résout alors le paradoxe du menteur en mettant en évidence que la proposition 2 est une affirmation de la proposition 1. II convient en outre de noter la permanence, en creux, de l'algèbre binaire; car la théorie des types logiques, en reposant sur la notion d'appartenance, n'offre que deux alternatives : soit l'objet appartient à un type, soit il ne lui appartient pas.

Dans l'introduction de Cybernetics, Wiener (2014a, p. 71) n'hésite pas à voir dans " l'influence de la logique mathématique un élément récurrent dans l'histoire de la cybernétique »; plus particulièrement, il se sert des types logiques de Russell pour conceptualiser le processus de la rétroaction :

Cette forme d'apprentissage est sans aucun doute une rétroaction, mais de niveau supérieur, celui des stratégies et non des actions élémentaires. Par rapport aux rétroactions plus basiques, elle n'est pas de ce que Russell appellerait du même "type logique". (Wiener, 2014b, p. 90)

La référence aux types logiques de Russell permet alors à Wiener de distinguer deux formes de rétroaction :

Comme je l'ai dit, la rétroaction est la commande d'un système au moyen de la réintroduction, dans ce système, des résultats de son action. Si ces résultats ne sont utilisés que comme données numériques pour l'examen et le réglage du système, nous obtenons la rétroaction simple que connaissent bien les automaticiens. Si, par contre, l'information portant sur l'action effectuée est capable de modifier la méthode générale et le modèle de celle-ci, nous disposons d'un processus que l'on peut bien nommer apprentissage. (Wiener, 2014b, p. 93)

Nous sommes à ce point, ici-même, au nœud de l'affaire : Wiener est l'un des savants ${ }^{1}$ qui opère la conversion et la concrétisation de la révolution logique propositionnelle. Avec lui, l'univocité prend la forme épistémologique de la rétroaction (et de ses synonymes: régulation, contrôle, feedback en anglais)

\footnotetext{
${ }^{1}$ Warren McCulloch et Walter Pitts (1943), scientifiques ayant pris part à la cybernétique naissante, s'emparèrent de la logique booléenne pour penser, dans un article célèbre, le « neurone formel ».
} 


\section{D'univocité en rétroaction :}

\section{la critique de la cybernétique dans la pensée de Heidegger}

qui s'incarne dans de multiples savoirs et dispositifs: les sciences cognitives, l'intelligence artificielle, les nouvelles théories de la communication (école dite de Palo Alto), le management contemporain, etc., qui tous reposent sur le même modèle formel d'apprentissage. L'information, puisqu'elle est abstraite et logique, est délivrée de la matière et peut donc indépendamment circuler dans différents supports: le corps humain, le psychisme, l'entreprise, la société, l'ordinateur. C'est bien la raison pour laquelle Jean-Pierre Dupuy (1999) et Céline Lafontaine (2004), dans leurs ouvrages respectifs, ont pu faire état de l'abondante descendance de la cybernétique.

Le processus de rétroaction se trouve au cœur des écrits des années 1960 au cours desquelles Heidegger cite et commente la cybernétique :

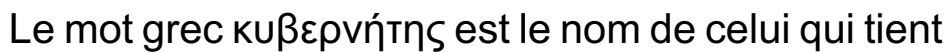
les commandes. Le monde scientifique devient monde cybernétique. Le projet cybernétique du monde suppose, dans sa saisie préalable, que la caractéristique fondamentale de tous les processus calculables du monde soit la commande. La commande d'un processus par un autre est rendue possible par la transmission d'une information. Dans la mesure où le processus commandé renvoie des messages à celui qui le commande et ainsi l'informe, la commande a le caractère de la rétroaction des informations. (Heidegger, 1983, p. 88)

Notons que, tout comme Wiener, Heidegger associe immédiatement la commande à la rétroaction. Les choses se précisent encore dans le paragraphe suivant; le philosophe introduit ici une nouvelle notion: «[...] la circularité de la régulation est le caractère fondamental du monde que projette la cybernétique ». Le caractère circulaire tient à la boucle de rétroaction qui reconduit toute action engagée, par le biais d'un traitement de l'information, à la commande initiale : voilà ce qui précisément se nomme régulation. Et tout comme Wiener parlait d' " autoreproduction " puis d' " auto-organisation " dans les deux derniers chapitres de La cybernétique, Heidegger conditionne très justement la possibilité de l'autorégulation et de l'automatisation à la circularité de la rétroaction. 
Le philosophe franchit à ce moment un pas supplémentaire dans son argumentation. Déroulant la définition que nous citions plus haut, Heidegger (1983, p. 88) écrit que « dans la représentation du monde par la cybernétique, la différence entre les machines automatiques et les êtres vivants est abolie. Elle est neutralisée par le processus de l'information qui ne fait pas de différence ». L'information est un équivalent général, au sens où l'argent l'était dans la philosophie marxienne (Marx, 1985, p. 68) : « La pensée calculante s'oblige elle-même à l'obligation de tout maîtriser à partir de la logique de sa manière de faire ", écrit ainsi Heidegger (1968, p. 80) en 1929 dans Qu'est-ce que la métaphysique?

Toutefois, dans la programmation de ce continuum " machine - animal - homme », nous pourrions croire que le dernier terme semble faire obstacle, et qu'il pourrait jouer le rôle d'un «facteur de perturbation »: «[...] une régulation de l'existence humaine n'est pas encore accomplie à l'heure actuelle » (Heidegger, 1983, p. 88). Pourtant, si la possibilité technique n'est pas encore d'actualité à l'époque d'Heidegger, ce dernier comprend que toutes les catégories ontologiques et épistémologiques sont désormais instituées pour laisser place à ce type de projet. II peut ainsi écrire :

Mais tout récemment la science a aussi pris possession de ce champ [celui de l'action] de l'existence humaine. Elle entreprend l'exploration et la planification, rigoureusement méthodique, de l'avenir possible de l'homme agissant. Elle prend en compte les informations sur ce qui est planifiable de l'homme. Cette sorte d'avenir est le futur pour le Logos qui, en tant que futurologie, se subordonne à la victoire de la méthode sur la science. La parenté de cette très récente discipline scientifique avec la cybernétique est évidente. (Heidegger, 1983, p. 89)

En d'autres termes, cela signifie qu'il n'y a pas d'un côté les projets de développement de l'intelligence artificielle, de l'autre les études sur la cognition, et encore ailleurs les applications sociales de la cybernétique. Tout cela ne fait qu'un, tout cela constitue une unité de vues, de sens et d'actions car, au fond, "société veut dire : société industrielle » (Heidegger, 1983, p. 89), ce qui signifie plus précisément que «les rapports de 


\section{D'univocité en rétroaction :}

\section{la critique de la cybernétique dans la pensée de Heidegger}

l'homme au monde, et, avec eux, la totalité de l'existence sociale de l'homme sont enclos dans le domaine où la science cybernétique exerce sa maîtrise » (Heidegger, 1983, p. 89). Cela signifie que l'homme ne se laisse plus déterminer par la parole comme animal métaphysique et politique, mais qu'il se fond, se confond, dans la circularité des boucles d'asservissement qui régulent l'ensemble de son existence.

\section{Cybernétique, dé-médiation et immédiation}

Ce que l'on désigne aujourd'hui par l'acronyme NBIC (pour Nanotechnologies, Biotechnologies, Informatique, sciences Cognitives), et qui se caractérise par une convergence et une intégration des différents savoirs et techniques, est déjà contenu en germe dans la cybernétique. Ce n'est en effet qu'à la stricte condition que l'ensemble des connaissances se formulent dans une même langue, s'énoncent à travers les mêmes catégories et se pensent sous le même procès de modélisation - la boucle de rétroaction - qu'une convergence des savoirs devient possible. Telle fut bien l'œuvre de la cybernétique qui, quoique son nom disparût de l'espace public dès les années 1950, n'en infiltra pas moins la quasi-totalité des disciplines scientifiques; citons ici cette longue énonciation de Jean-Pierre Dupuy :

[La cybernétique] aura, en vrac et sans souci d'exhaustivité : introduit la conceptualisation et le formalisme logico-mathématique dans les sciences du cerveau et du système nerveux; conçu l'organisation des machines à traiter l'information et jeté les fondements de l'intelligence artificielle; produit la "méta-science" des systèmes, laquelle a laissé son empreinte sur l'ensemble des sciences humaines et sociales, de la thérapie familiale à l'anthropologie culturelle; fortement inspiré des innovations conceptuelles en économie, recherche opérationnelle, théorie de la décision et du choix rationnel, théorie des jeux, sociologie, sciences du politique et bien d'autres disciplines; fourni à point nommé à plusieurs "révolutions scientifiques" du XXe siècle, très diverses puisqu'elles vont de la biologie moléculaire à la relecture de Freud par Lacan, les métaphores dont elles avaient besoin pour marquer leur rupture par 


\section{Baptiste RAPPIN}

rapport à des paradigmes établis. (Dupuy, 1999, p. 34-

35)

Comment une telle paternité fut-elle rendue possible? Tout d'abord par des circonstances historiques : dès 1942, puis entre 1946 et 1953, se réunirent, à l'initiative de la fondation Joshua Macy, des scientifiques d'horizons divers et variés: Norbert Wiener (mathématicien), Warren McCulloch (neurologue), John von Neumann (mathématicien, informaticien), Walter Pitts (mathématicien), Gregory Bateson et son épouse Margaret Mead (anthropologues), Kurt Lewin et son disciple Alex Bavelas (psychologie sociale), Paul Lazarsfeld (sociologue), etc. La liste n'est certes pas exhaustive, mais pointe en direction de l'essentiel : l'effort de communication entre disciplines, à l'origine des mouvements d'interdisciplinarité tant prisés aujourd'hui, a pour objectif la formulation d'une méta-science qui donnerait à chacune de ses sœurs régionales ses fondements et ses assises. Ainsi la cybernétique n'est-elle pas qu'une rencontre, elle est aussi, et pleinement, un projet et un contenu.

Ruse de l'histoire oblige, ces brillants chercheurs qui souhaitaient communiquer entre eux firent de la communication le fondement d'une nouvelle ère scientifique, comme le résume Michel Serres (1974, p. 41) en une courte phrase : "Le savoir contemporain, dans son ensemble, est une théorie de la communication ». Ce qui signifie, si l'on se remémore les développements précédents, que l'univocité de la logique propositionnelle et sa mise en œuvre dans la boucle de rétroaction servent d'atomes théoriques aux nouveaux savoirs.

Heidegger avait, de façon très nette, identifié l'unification des sciences par la cybernétique. Comment expliquer ce célèbre passage de l'entretien au journal Der Spiegel ? « Spiegel : Et qui prend maintenant la place de la philosophie ? Heidegger: La cybernétique » (Heidegger, 1995, p. 262). Voici la réponse, formulée comme une anticipation : " II n'est pas besoin d'être prophète pour reconnaître que les sciences modernes dans leur travail d'installation ne vont pas tarder à être déterminées et pilotées par la nouvelle science de base, la cybernétique » (Heidegger, 1990c, p. 284). Si la philosophie était traditionnellement considérée comme la reine des sciences, garantissant à ces dernières un fondement stable à l'image de l'arbre que Descartes utilise dans la lettre-préface aux Principes de la Philosophie (Descartes, 1999), ce rôle est désormais bel et 


\section{D'univocité en rétroaction :}

\section{la critique de la cybernétique dans la pensée de Heidegger}

bien révolu: la cybernétique a détrôné la métaphysique et assure désormais l'unité des sciences non plus en référence à un principe, mais en vertu de la portée opérationnelle et de l'efficacité technique des modèles déployés :

"Théorie" signifie maintenant: supposition de catégories auxquelles n'est accordée qu'une fonction cybernétique, toute signification ontologique leur étant déniée. Le caractère opératoire et la référence au modèle de la pensée représentative et calculante en sont venus à régner en maîtres. (Heidegger, 1990c, p. 285)

La cybernétique assure son hégémonie, car elle « imprime, non seulement aux sciences mais à toute activité humaine, le caractère de la planification et du guidage " (Heidegger, 1990d, p. 15). La planification et le guidage ne renvoient à rien d'autre qu'à la commande et au contrôle, termes par lesquels Norbert Wiener définit lui-même la cybernétique, dont l'étymologie grecque, kybernetès, renvoie directement au pilotage; en d'autres termes, la boucle de rétroaction (que l'on pourra encore nommer "apprentissage " ou "amélioration continue " et même "temps réel ») devient le modèle dans lequel doit se couler l'ensemble des activités sociales. De ce point de vue, Éric Sadin (2015) est justifié à écrire que "tout être se situe désormais au sein d'une boucle, engendrant des myriades de codes et recevant des informations de tous ordres [...] » (p. 133).

C'est dire qu'il s'agit là non pas seulement d'une révolution scientifique, pour reprendre l'expression de Thomas Kuhn, mais bien d'une mutation métaphysique et anthropologique fondamentale; la cybernétique, qui unifie les sciences en leur conférant un caractère immédiatement opératoire, définit simultanément les nouveaux principes directeurs de la vie sociale :

"Cette science correspond à la détermination de I'homme comme être dont l'essence est l'activité en milieu social. Elle est en effet la théorie qui a pour objet la prise en main de la planification possible et de l'organisation du travail humain » (Heidegger, 1990c, p. 285).

Ce qui signifie d'une part que la cybernétique constitue la matrice à partir de laquelle s'est déployé le management 


\section{Baptiste RAPPIN}

contemporain (Rappin, 2014, 2018), mais également que l'ensemble des savoirs constitués à partir des catégories de la cybernétique n'ont pour visée que la seule efficacité de l'action.

On peut prolonger, voire compléter, les analyses de Heidegger, qui relèvent de l'ontologie fondamentale, en envisageant cette fois-ci le champ socio-historique: en imprimant le modèle de l'organisation (définie comme boucle de rétroaction) à l'ensemble des activités humaines, la cybernétique, et la logique calculatoire et algorithmique qui la sous-tend, se trouve directement à l'origine du mouvement général de désinstitutionnalisation. Illustrons en premier cette thèse par deux citations. La première se lit sous la plume de Steward Brand, dont Fred Turner (2013) a montré l'importance décisive dans la constitution de l'utopie numérique :

"Qu'ils le veuillent ou non, les ordinateurs arrivent chez les gens, c'est une bonne nouvelle, la meilleure peut-être depuis les drogues psychédéliques [...]. Ils sont fabriqués par et pour des révolutionnaires qui veulent désinstitutionnaliser la société et donner du pouvoir aux individus » (Brand, cité dans Sadin, 2015, p. 54).

On découvre la seconde en lisant Peter Drucker (1992), un gourou de la gestion à qui l'on doit, entre autres, la gestion par objectifs directement inspirée de la cybernétique :

La société, la communauté et la famille sont toutes des institutions conservatrices. Elles essayent de maintenir la stabilité et de prévenir, à tout le moins de ralentir, le changement. Mais l'organisation moderne est un déstabilisateur [...]. Et elle doit être organisée en vue de l'abandon systématique de tout ce qui est établi.

Ces exemples nous conduisent à émettre l'hypothèse suivante: la cybernétique développe un projet proprement révolutionnaire de destitution généralisée (Rappin, 2019). En effet, toutes les institutions, qu'elles soient culturelles comme la famille, ou politiques comme le droit, représentent des médiations inutiles entre les individus; en tant que telles, elles sont en effet à la fois aliénantes, en ce qu'elles privent l'être humain d'un accès direct à autrui et au réel, et inefficaces car elles ralentissent le circuit qui part de soi et parvient à l'altérité 


\section{D'univocité en rétroaction :}

\section{la critique de la cybernétique dans la pensée de Heidegger}

en empruntant le chemin détourné d'un tiers. Dans un monde univoque gouverné par la logique du calcul algorithmique, dans lequel la déduction se substitue à la symbolisation, les individus sont sommés de s'adapter en temps réel aux conditions de leur milieu, ils sont contraints d'interagir directement avec autrui, sur le mode de l'ajustement mutuel, évoluant ainsi dans un plan horizontal d'immanence au sein duquel logique algorithmique et logique contractuelle se renforcent l'une l'autre.

\section{Conclusion : "Le fantôme d'un langage pur » (Merleau- Ponty, 1969, p. 7)}

On pourrait croire que cette étude ne concerne que les seuls spécialistes de l'œuvre de Martin Heidegger, ou alors qu'elle s'adresse à tous ceux qui affirment que le Gestell, cet ensemble de dispositifs techniques visant à rendre l'étant disponible dans son intégralité, est une catégorie par trop englobante, par trop massive, pour pouvoir offrir un regard analytique et précis sur la société industrielle. S'il est vrai que beaucoup découvriront, à travers notre article, que Heidegger se tint à l'écoute des avancées scientifiques de son temps et qu'il intégra ces dernières à son système de pensée, l'apport de notre travail réside, nous semble-t-il, dans l'exposé de la généalogie de la logique algorithmique que le philosophe met à jour. En quelque sorte, nous autres contemporains qui sommes surpris, voire stupéfaits par la célérité du développement des NBIC, retrouvons par ce geste la profondeur historique et métaphysique nécessaire à la compréhension des temps présents.

La logique algorithmique, c'est-à-dire l'enchaînement sans fin de calculs, s'enracine dans la tradition métaphysique occidentale, et plus particulièrement dans le principe de raison qui reçut sa formulation avec Leibniz : ce dernier nourrissait en effet le rêve d'une langue intégralement formelle, entièrement formalisée, soustraite à l'impureté des analogies et des équivocités. Ce projet connut une avancée décisive avec l'algèbre de Boole et la logique propositionnelle moderne qui conférèrent à l'univocité la langue qui lui sied. C'est cette même univocité qui, par l'intermédiaire de la théorie des types de Russell, fonde la thématisation cybernétique de l'organisation comme boucle de rétroaction: dans ce cadre, toute action se 


\section{Baptiste RAPPIN}

trouve, par l'instauration de processus de contrôle, ramenée à la finalité du système. L'immédiateté recherchée conduit alors à l'immédiation : à un court-circuit général de toutes les formes de médiation, et en premier lieu des institutions politiques qui garantissaient pendant la modernité l'espace public indispensable à la vie commune.

C'est en cela que le détour par l'œuvre de Heidegger nous paraît primordial: si les réflexions d'Antoinette Rouvroy et de Thomas Berns (2013) sur la gouvernementalité algorithmique et celles de Bernard Stiegler (2015) sur La société automatique sont tout à fait pertinentes, elles méritent d'être recontextualisées dans un temps long qui mène à prendre en compte ce principe : la transformation de la langue en information est bien la condition de possibilité ontologique de la logique algorithmique et de son déferlement. Et, en effet, « le rapport de l'homme à la langue est pris dans une mutation dont ne nous mesurons pas encore la portée »(Heidegger, 1990c, p. 63).

\section{Bibliographie}

Brown, S. D. et Lightfood, G. M. (1998). Insistent emplacement: Heidegger on the technologies of informing. Information Technology \& People, 11(4), 290-304.

Dastur, F. (2007). Heidegger. La question du Logos. Paris: Librairie Philosophique J. Vrin, «Bibliothèque des philosophies ».

Descartes, R. (1999). Les principes de la philosophie. Paris : Librairie Philosophique J. Vrin, «Bibliothèque des textes philosophiques ».

Drucker, P. (1992). The New Society of Organizations. Harvard Business Review, 70(5), 95-104. Article téléchargé sur le site de la revue le 31 mai 2017 : https://hbr.org/1992/the-newsociety-of-organizations.

Dupuy, J.-P. (1999). Aux origines des sciences cognitives. Paris : Éditions La découverte \& Syros, « Sciences Humaines et Sociales ".

Frege, F. (1971), Écrits logiques et philosophiques. Paris: Éditions du Seuil, « Essais ». 


\section{D'univocité en rétroaction :}

\section{la critique de la cybernétique dans la pensée de Heidegger}

Gaffiot, F. (2000). Dictionnaire Latin-Français. Paris : Hachette.

Hahn, H., Neurath, O. et Carnap, R. (1990). La conception scientifique du monde : le Cercle de Vienne. Dans R. Carnap, $\mathrm{O}$. Neurath, H. Hahn et M. Schlick, Manifeste du cercle de Vienne et autres écrits (p. 101-146). Paris : Librairie Philosophique J. Vrin, «Bibliothèque des textes philosophiques ".

Heidegger, M. (1962). Le principe de raison. Paris : Éditions Gallimard, « Tel ».

Heidegger, M. (1968). Questions I et II. Paris: Éditions Gallimard, « Tel ».

Heidegger, M. (1971a). Qu'est-ce qu'une chose? Paris: Éditions Gallimard, "Tel ».

Heidegger, M. (1971b). Nietzsche II. Paris: Éditions, Gallimard, "Bibliothèque de Philosophie ".

Heidegger, M. (1976). Acheminement vers la parole. Paris : Éditions Gallimard, « Tel ».

Heidegger, M. (1983). La provenance de l'art et la destination de la pensée. Dans M. Haar (dir.), Martin Heidegger (p. 84-93). Paris : Éditions de l'Herne, "Cahiers de l'Herne ».

Heidegger, M. (1986). Être et Temps. Paris: Éditions Gallimard, "Bibliothèque de philosophie ".

Heidegger, M. (1990a). Questions III et IV. Paris : Éditions Gallimard, «Tel ».

Heidegger, M. (1990b). Langue de tradition et langue de pensée. Bruxelles, Belgique: Éditions Lebeer-Hossmann, «Philosophiques ".

Heidegger, M. (1990c). Questions III et IV. Paris : Éditions Gallimard, « Tel ».

Heidegger, M. (1990d). L'affaire de la pensée (Pour aborder la question de sa détermination). Mauzevin: Trans-EuropExpress.

Heidegger, M. (1995). Écrits politiques. 1933-1966. Paris: Éditions Gallimard, « Bibliothèque de Philosophie ».

Heidegger, M. (1999). Qu'appelle-t-on penser? Paris: Presses Universitaires de France, "Quadrige ». 


\section{Baptiste RAPPIN}

Introna, L. D. (2001). The question concerning information technology: thinking with Heidegger on the essence of information technology. Dans J. D. Haynes (dir.), Internet Management Issues: A Global Perspective (p. 220-234). Hershey, États-Unis : IGI Global.

Janicaud, D. (2001). Heidegger en France. Tome 2: entretiens. Paris: Albin Michel, Hachette Littératures, « Pluriel Philosophie ».

Lafontaine, C. (2004). L'empire cybernétique. Des machines à penser à la pensée de la machine. Paris : Éditions du Seuil.

Leibniz, G. W. (1995). Discours de métaphysique suivi de Monadologie. Paris : Éditions Gallimard, "Tel ».

McCulloch, W. et Pitts, W. (1943). A Logical Calculus of the Ideas Immanent in Nervous Activity. Bulletin of Mathematical Biophysics, 5, 115-133.

Marx, K. (1985). Le capital. Livre I, sections I à IV. Paris : Flammarion, « Champs ».

Merleau-Ponty, M. (1969). La prose du monde. Paris: Éditions Gallimard, « Tel ».

Rappin, B. (2014). Au fondement du Management. Théologie de l'Organisation, Volume 1. Nice : Éditions Ovadia, "Chemins de pensée ".

Rappin, B. (2015). Heidegger et la question du Management. Cybernétique, Information et Organisation à l'époque de la planétarisation. Nice : Éditions Ovadia, «Chemins de pensée ».

Rappin, B. (2018). De l'exception permanente. Théologie de l'Organisation, Volume 2. Nice: Éditions Ovadia, «Les carrefours de l'être ".

Rappin, B. (2019). De la révolution managériale au management révolutionnaire. Le Philosophoire, 51, 187-202.

Rouvroy, A. et Berns, T. (2013). Gouvernementalité algorithmique et perspectives d'émancipation. Réseaux, 177, 163-196.

Sadin, E. (2015). La vie algorithmique. Critique de la raison numérique. Paris : Éditions L'Échappée. 


\section{D’univocité en rétroaction :}

\section{la critique de la cybernétique dans la pensée de Heidegger}

Serres, M. (1974). Hermès III. La traduction. Paris : Éditions de Minuit, "Critique ».

Stiegler, B. (2015). La société automatique. 1 : L'avenir du travail. Paris : Fayard.

Turner, F. (2013). Aux sources de l'utopie numérique. De la contre-culture à la cyberculture: Steward Brand, un homme d'influence. Caen : C\&F Éditions.

Turing, A. (1995). La machine de Turing. Paris : Éditions du Seuil, « Points Science ».

Wiener, N. (2014a). La cybernétique. Information et régulation dans le vivant et la machine. Paris : Éditions du Seuil, "Sources du savoir $»$.

Wiener, N. (2014b). Cybernétique et société. L'usage humain des êtres humains. Paris : Éditions du Seuil, "Sciences" .

Wittgenstein, L. (1993). Tractatus logico-philosophicus. Paris : Éditions Gallimard, « Tel ». 